\title{
Introduction éditoriale
}

\author{
Martine Béland
}

Ce numéro non thématique de la revue PhoenEx montre une fois de plus la variété des intérêts de recherche de ceux qui ont fait de la philosophie continentale leur champ de prédilection. Notre section «Varia» présente en effet des articles de chercheurs canadiens, américains, québécois et français, qui portent sur la phénoménologie des mathématiques, du handicap ou du genre, ou sur des penseurs importants pour les études sur le corps et sur la liberté (Beauvoir, Levinas, Barbaras, Grimaldi). Cinq notes de lecture proposent ensuite des discussions d'ouvrages récents sur le néokantisme de Vaihinger, les essais sur la photographie de Kracauer, la pensée de Jean-Luc Nancy, l'éthique de la psychanalyse de Lacan et l'ontologie merleau-pontienne. Nous remercions tous les auteurs ayant contribué à ce numéro d'avoir confié leurs travaux à la revue PhoenEx!

\section{Philosophie et éducation : un dialogue de sourds?}

En guise d'ouverture à ce numéro, nous avons regroupé trois textes qui portent plus précisément sur la philosophie et l'éducation. Ce thème fait directement écho aux discussions actuelles sur l'avenir de l'éducation supérieure dans les pays occidentaux. La question est posée autant aux États-Unis qu'au Québec ou en Europe : comment le diktat de la croissance économique et les politiques néolibérales peuvent-elles être réconciliées avec l'idéal humaniste qui a modelé nos institutions d'enseignement supérieur? Comment maintenir une ouverture de ces institutions au plus grand nombre de jeunes possibles dans un contexte où la part des fonds publics allouée à l'enseignement diminue sans cesse, autant en éducation supérieure qu'en éducation primaire et secondaire? Comment la formation humaniste (qu'on l'appelle formation générale au Québec ou liberal-arts education aux États-Unis) peut-elle remplir son mandat, conserver sa mission et maintenir son territoire en éducation, face au développement frénétique, par les universités d'ici et d'ailleurs, de diplômes courts, plus «techniques » et axés vers l'insertion des jeunes dans le marché du travail? 
Ces questions, discutées par des professeurs des milieux universitaires et collégiaux ainsi que par divers acteurs de la société civile, sont de plusieurs tribunes depuis maintenant quelques années, et ponctuent régulièrement les débats dans les journaux et revues et au sein des milieux académiques et culturels. Aux États-Unis, on a pu lire récemment un essai de William Deresiewicz sur les difficultés énormes auquel fait face le mandat initial des collèges américains. Sans le savoir, le constat sur la situation des liberal arts colleges aux États-Unis se fait l'écho direct des craintes qui animent les milieux des collèges d'enseignement général et professionnel au Québec. Une journée de conférences et de réflexions fut tenue en septembre 2015 à la Grande bibliothèque (Montréal) sur des questions qui occupaient récemment les pages de la revue Liberté : son numéro d'automne 2014, sous le titre Ministère de la Formation, portait sur l'éducation à l'ère du management. L'essayiste John Cassidy posait lui aussi la question d'une manière on ne peut plus claire dans les pages du New Yorker : "What's the real value of higher education? » Son article se terminait sur une remarque commune à presque tous les auteurs qui écrivent maintenant sur ce thème : «Being more realistic about the role that college degrees play [...] could also help us appreciate the actual merits of a traditional broad-based education, often called a liberal-arts education, rather than trying to reduce everything to an economic costbenefit analysis. » (Cassidy 84)

Il est évident que le thème «Philosophie et éducation », de par son caractère actuel mais classique, et de par les liens manifestes et, semble-til, permanents entre la pratique de la philosophie et la pratique de l'enseignement, mérite bien sûr un éclairage particulier — il méritait même un numéro thématique entier! Pourtant, bien qu'il fut annoncé d'avance, ce thème - il nous faut l'avouer à nos lecteurs — n'a donné lieu à à peu près aucune soumission... Doit-on, du coup, céder à la tentation de formuler un diagnostic pessimiste, voire cynique, quant à l'intérêt réel que pose la question de l'éducation pour la recherche en philosophie ou que pose la question de la philosophie pour la recherche en éducation?

Pour lancer cette question et «mettre la table », nous avons invité le philosophe Normand Baillargeon — professeur en éducation à l'UQÀM, auteur prolifique et chercheur réputé, des deux côtés de l'Atlantique, pour sa connaissance de la philosophie de l'éducation et ses nombreuses contributions à ce champ trop peu foulé - à proposer une réflexion issue de sa longue expérience de chercheur et de professeur, laquelle est sans conteste enrichie, d'une part, par sa double spécialisation en philosophie et en éducation, et, d'autre part, par sa connaissance de la tradition analytique qu'il fait dialoguer avec le corpus dit continental. Nous sommes heureux qu'il ait accepté notre invitation et qu'il livre aux 
lecteurs de PhoenEx son texte intitulé «La philosophie de l'éducation en science de l'éducation : un témoignage ».

Nous proposons ensuite un article de Rebecca Glenn Scott, doctorante à l'Université Loyola (Chicago) et membre du comité exécutif de l'American Association of Philosophy Teachers. Son article, «The Fertility of Dialogue: Levinas and Plato on Education », porte sur la critique levinassienne de l'éducation socratique telle que celle-ci se déploie dans la maïeutique. L'auteure veut montrer, contre Levinas, que la maïeutique demande une réelle prise en compte de l'altérité, et qu'en ce sens, une conception de l'éducation comme pratique maïeutique peut et doit avoir des effets positifs sur la pédagogie actuelle. Le concept, la pratique et l'éthique de l'enseignement sont donc au cœur de son propos. Son article nous semblait tout indiqué pour cette section sur la philosophie et l'éducation, puisque l'auteure espère ainsi réorienter la manière dont on comprend, aujourd'hui, ce qu'est la pédagogie philosophique.

Pour clore notre section sur la philosophie et l'éducation, nous proposons une lecture de l'ouvrage collectifQu'appelle-t-on un séminaire? La pédagogie heideggerienne, par Peter Odabachian, professeur de philosophie au Cégep Édouard-Montpetit. Ce livre sous la direction de Christophe Perrin, spécialiste de Heidegger, est issu d'un séminaire tenu au Collège international de philosophie (Paris) au cours de l'année 2011-2012. Les contributions, provenant toutes de jeunes chercheurs qui travaillent en philosophie allemande ou en phénoménologie, se penchent sur les divers sens de la pédagogie heideggerienne et sur différents aspects de cette pratique de la philosophie dans le cadre du séminaire - tels que le rythme, l'écoute ou le style — ou encore sur son contexte et ses modèles. En plus de l'originalité manifeste de ce thème dans le cadre des études heideggeriennes, le grand mérite de cet ouvrage est de rappeler — puisqu'il semble bien qu'il le faille! — qu'un philosophe, de nos jours, est presque toujours aussi un professeur, c'est-àdire quelqu'un qui enseigne et qui a donc adopté (parfois consciemment, parfois tacitement) une pratique pour former autrui.

Cette toute petite section sur la philosophie et l'éducation sera donc partie du pronostic établit par Normand Baillargeon sur l'effet délétère du désintérêt pour la philosophie de l'éducation (désintérêt manifeste autant du côté des philosophes que de celui des spécialistes des sciences de l'éducation, précise Baillargeon), pour aboutir au constat, issu de la discussion menée par Peter Odabachian, qu'il est bien rare que la recherche en philosophie se penche sur l'une de ses pratiques les plus courantes, à savoir l'enseignement de la philosophie. Entre les deux, on sera passé au moins par une tentative, faite par Rebecca Glenn Scott, pour donner un nouveau souffle à une certaine pédagogie philosophique. Nous espérons que nos lecteurs trouveront ici l'occasion de se pencher plus 
profondément sur le sens pédagogique de la pratique de la philosophie et sur la dimension philosophique de l'éducation.

Nous souhaitons, pour terminer, remercier tous les chercheurs et professeurs qui ont évalué anonymement les articles soumis à la revue en 2014-2015, ainsi que les maisons d'édition et les distributeurs qui nous ont envoyé des exemplaires de presse des livres recensés. En tant que rédactrice en chef, je souhaite aussi remercier l'équipe éditoriale de PhoenEx, sans laquelle la production des numéros de la revue serait tâche impossible.

\section{Textes cités}

CAssidy, John, "College Calculus. What's the Real Value of Higher Education? », The New Yorker, 7 sept. 2015, p. 80-84.

DeresiewICZ, William, «The Neoliberal Arts. How College Sold its Soul to the Market », Harper's Magazine, sept. 2015, p. 25-32.

PERRIN, Christophe (dir.), Qu'appelle-t-on un séminaire? La pédagogie heideggerienne, Bucarest, Zeta Books, 2013.

Revue Liberté, $\mathrm{n}^{\circ} 305$ : Ministère de la Formation. L'éducation à l'ère du management, automne 2014. 